Aas der Kgl. Universitätsklinik für Hautkrankheiten in Kiel. (Direktor: Prof. Dr. Klingmäller.)

\title{
Über das Schicksal hereditär syphilitischer Kinder [Lues hereditaria tarda?].
}

\author{
Von \\ Privatdozent Dr. med. Fr. Bering, \\ Oberarzt der Klinik.
}

Welander bat das große Verdienst, mit Nachdruck auf die über längere Zeit ausgedehnte Beobachtung und Behandlung kongenital syphilitischer Kinder aufmerksam gemacht zn haben. Seine Ideen bat er zu verwirklichen gesucht durch Errichtung des dem Krankenhaus St. Göran angegliederten Asyls "lilla hemmet".

Zun Schaden der vielen unglücklichen Kinder hat die Anregung nur wenig oder gar keine Nachahmung gefunden.

In solchen Anstalten würde man auch wertvolle Aufschlüsse erhalten über den Verlauf der Syphilis in den ersten Lebensjahren. Wir verlieren ja leider jetzt die Kinder schon bald nach der Geburt, wenn keine Zeichen der Syphilis bestehen, oder doch wenigstens nach dem glücklichen Überstehen der ersten Symptome aus den Augen. Sic suchen den Arzt erst wieder auf, wenn neue Erscheinungen auftreten. Sie selbst und besonders ihre Angehörigen erinnern sich kaum noch der früheren Krankheit, so dab man sie denjenigen Fällen hereditärer Lues zurechnet, die bisher scheinbar gesund, erst lange Zeit nach der Geburt, oft nach Jahren, an den Symptomen ihres ererbten Leidens erkranken.

Wir baben nun Gelegenheit gehabt, eine Reihe solcher Fälle längere Zeit zu beobachten, über welche ich im folgenden 
berichten möchte. Man köunte geneigt sein, diese Fälle als Syphilis hereditaria tarda zu bezeichnen.

Unter Syphilis hereditaria tarda versteht man diejenige Form der Syphilis hereditaria, bei welcher das Kind längere Zeit nach der Gebut, bis zur Pubertät und sogar noch über diese hinaus, latent syphilitisch sein kaun, um dann an Erscheinungen zu erkranken, die der Spätperiode der Syphilis angehören.

Die Aufstellung dieser besonderen Art wird von einer Reihe von Syphiliskennern als nicht berechtigt angesehen. Die hauptsächlichsten Ansichten einiger Forscher seien hier angefiihrt. Lew in (Berl. klin. Wochenschrift 1876) betrachtet sie als den "Ausbruch eines bis dahin im Organismus schlummernden Keimes". Virch ow nimmt an, daß in den inneren Organen Infektionsherde verborgen sein könnev. „Ehe diese andere Teile in der Art infizieren, dab die Eruptionen sichtbar, äuBerlich werden, darüber können Tage, Wochen, ja selbst Jahre vergehen." Die L. b. t. muß in gewissen Krankheitsherden schon bei der Geburt vorbanden sein. Auch $\mathrm{Z}$ e issl nimmt eine angeerbte latente Syphilis an, welche erst später, zuweilen erst im Jünglingsalter zum Ausbruch kommt. Besonders eingebend hat sich A. Fo urnier mit dieser Frage beschäftigt. (La Syphilis héréditaire tardive, Paris 1886 und L'hérédité syphilitique, Paris 1891). Er rechnet nicht nur die Fälle hinzu, in denen die syphilitischen Prozesse im späteren Alter überhaupt zum ersten Male einsetzten, sondern auch jene, in denen "kein verdächtiges Symptom den Argwohn der Eltern oder den Verdacht des Arztes erregte". Es können also schon in "früher Kindheit" Erscheinungen vorausgegangen sein; die späteren sind dann als Rezidive aufzufassen. Auch Heinrich (Archiv f. Derm. u. Syphilis, Bd. XC) ist der Ansicht, „daB in jedem Falle von tardiver Erhsyphilis dem späteren Ausbruch Vorläufer während des intra- und in der allerersten Zeit des extrauterinen Lebens vorausgegangen sind und daß der spätere, scheinbar erste manifeste Ausbruch nur das durch irgend einen äußeren Zufall (vielleicht ein Trauma) verursachte Wiederaufflackern der Tätigkeit der an irgend einer Stelle des Körpers schlummernden Spirochaeten bedeutet." 
Andere Autoren sprechen sich gegen das Vorhandensein der Syphilis hereditaria tarda aus; wie z. B. W olf (Über Syphilis hereditaria tarda), Roussel (Syphil. hérédit. tardive), Neumann (Nothnagel Bd. 23). Bei Kassowitz (Die Vererbung der Syphilis) waren in keinem der von ibm beobachteten Fälle die ersten Symptome der bereditären Lues später als im 3. Monat zum Ausbruch gekommen. Neisser gibt theoretisch die Möglichkeit der Syphilis hereditaria tarda zu; glaubt aber nicht an ihr tatsächliches Vorkommen.

Folgende Überlegungen sind nach unserer Ansicht für die Auffassung des Verlaufes der Syphilis hereditaria und ihrer tardiven Form wesentlich. Die Syphilis ist eine polymorphe Krankheit und die Polymorphie erklärt den so auBerordentlich verschiedenen Verlauf der Syphilis überhaupt. Es besteht wohl darüber kein Zweifel, daß die ehemalige Schematisierung, nämlich die Einteilung in primäre, sekundäre und tertiäre Lues völlig veraltet ist und höchstens noch aus didaktischen Gründen gelegentlich erwähnt werden darf. Die Abtrennung der einzelnen Formen stößt in der Praxis auf so große Schwierigkeiten, daß man selbst die Einteilung in Früh- und Spätformen manchmal nicht machen kann. Dieses ist noch schwieriger, seit wir wissen, daß auch die Spätsyphilide ihre Entstehung der Anwesenheit von Spirochaeten verdanken und nicht auf die Wirkung unbekannter Toxine zurückzuführen sind.

Doch ganz abgesehen davon kommt der Verla uf der Lues bei den einzelnen Individuen hinzu. Und auch hier sehen wir, daß die Lues in den weitesten Grenzen Erscheinungen hervorrufen kann von den harmlosesten spontan heilenden Fällen bis zu den schwersteu Zerstörungen. Ferner erlaubt der vorherige Verlauf der Lues keinerlei Schlüsse auf die Zukunft. Jeder weiß, daß sich die Prognose in jedem Augenblick nach der guten, oder, was leider häufiger eintritt, nach der schlechten Seite hin ändern kann.

Berücksichtigen wir diese Überlegungen, so wird sich auch für die hereditäre Lues eine grobe Verschiedenheit unter den einzelnen Fällen ergeben.

Diese Verschiedenheit hängt nach unserer Meinung mehr ab von der Zeit der Infeltion als der Menge der Erreger, 
welche übertragen werden. Je frühzeitiger in utero die Infektion stattfindet, um so früher stirbt der Foet oder das Kind; je später, um so günstiger ist die Prognose für die Erhaltung des Kindes. Der Zeitpunkt der Infektion in utero scheint uns auch insofern wesentlich zu sein, ob bereits die foetalen Organe entwickelt oder noch unentwickelt sind. Macht der Foet eine Erkrankung durch vor der Ausbildung der betreffenden Organe, so wird selbstverständlich die Krankheit auf solche Organe einen anderen Einfluß ausüben, als wenn sie schon gewissermaßen fertig sind. Auf diese ungezwungene Weise erklären sich wohl am besten die Eigentümlichkeiten der Exscheinungen bei der hereditären Lues. Ein wichtiges Moment kommt freilich noch hinzu: die Lokalisation der Spirochaeten; sie wirkt, ob zufällig oder durch gewisse Ursachen (Traumen, Sekundärinfektion, sonstige Schwäche der betreffenden Organe usw.) herrorgerufen, mitbestimmend auf das Krankheitsbild ein.

Dadurch erklärt sich auch diese Verschiedenheit, daß Kinder entweder mit frisch syphilitischen Erscheinungen geboren werden od er scheinbar gesunde Kinder zur Welt kommen, welche unter Umständen erst nach langer Zeit (Jahren) syphilitische Symptome bekommen. $Z$ wis chen diesen beiden Formen stehen solche Fälle, welche mit den Zeichen (z. B. Narben) bereits abgelaufener Krankheitsprozesse geboren werden.

Wenn wir uns über diese Punkte klar sind, so dürfen wir die Syphilis hereditaria tarda $\mathrm{nicht}$ als einen besonderen Symptomenkomplex auffassen, sondern wir verstehen darunter solche Fälle, welche die Infektion in utero erwerben, die ersten Erscheinungen hier überstehen und nach verschieden langer Latenz (scheinbarer Heilung) von neuem und dann meist an Spätformen der Lues erkranken.

Derartige Fälle sind naturgemäß nicht häufig. Es erscheint mir daher berechtigt, über das Schicksal solcher Kranken zu berichten und festzustellen, welche Krankheitserscheinungen sie nach unseren Beobachtungen wenigstens im späteren Leben darbieten. Zunächst seien kurz die Krankengeschichten von 37 Fällen mitgeteilt.

1. E r win G., 10 Jahre alt. Eltern leben, gesund; ebenfalls 3 Geschwister völlig gesund; 1 Bruder kann schlecht sehen. Im 2. Lebens- 
ja hre soll langsame Erblindung eingetreten sein. Muskulatur kräftig; Ernährunggzustand gut. Innere Organe o. B. An r. Tibia Auftreibung in der Mitte; Exostose (Säbelscheidentibia). Langschädel; Stirn schmal. Lippenrot mit Rhagaden bedeckt. Zähne defekt; bröckelig; weit auseinanderstehend. Zervikal-Kubitaldrüsen erbsengroB, hart. Seroreaktion: negativ. Augen: Keratitis parencbymatosa.

2. A n n a J., 19 Jahre. Eltern leben; angeblich gesund. $3 \mathrm{Ge}$ schwister leben. 5 Geschwister als kleine Kinder gestorben; sie kann seit dem 5. Lebensjahr schlecht sehen; seit dem 4. schlecht hören. Sehr guter Ernährungszustand, Muskulatur schlaff. Keine Wachstumstörungen; säbelscheidenförmige Verkrümmung beider Tibien. Herz, Lunge, Leber gesund. Viereckiger Schädel mit Asymmetrie. Völlige Taubbeit links. Ohr groß, weit abstehend. Lippen tiefe radiare Narben. Sehr schöne Hutchinsonzähne. Hoher Gaumen. Diffuse Alopezie. Hautfarbe normal. Keine Narbon, keine Drüsen. Intelligenz mäßig. S.-R. negativ. Augen: Keratitis parenchymatosa.

3. M a r i e F., 12 Jahre. Vater und Mutter angeblich gesund, niemals krank gewesen. Keine Aborte oder Frühgeburten. 2. Kind ist ein völlig gesunder Knabe. Ende Januar 1910 Sehstörungen als erste Erscheinuugen. Guter Ernährungszustand. Geringe Asymmetrie des Schädels. Sattelnase. Zervikaldrüsen erbsengroß, hart. Angeblich zeitweilig Jucken in beiden Händen. Keratitis parenchymatosa. Wassermann positiv. Eine Schmierkur hat nur wenig geholfen. Macht jetzt Tuberkulinkur A. T., worauf geringe Besserung; däs Sehvermögen soll sich bessern.

4. Emil K., 17 Jahre alt. Über seine Eltern und Geschwister weiß E. keine Angaben zu machen. Er selbst erkrankte im Alter von 7 Jahren an Unterschenkelgeschwüren und erblindete im Alter von 9 Jahren innerhalb kurzer Zeit. Großer, kräftig gebauter Knabe in gutem Ernăhrungszustand. Innere Organe o. B. Tiefe radiäre Narben auf den Lippen. Asymmetrischer Schädel. Völiige Erblindung infolge doppelseitiger Keratitis parenchymatosa. Zervikal- und Kubitaldrüsen hart, fast bohnengroß. Typische Säbslacheidentibien. Vom 5. Februar 1909 bis 14. April 1909 in hiesiger Klinik: Damals waren beide Unterschenkel bedeckt mit zahlreichen, pfennig. bis 3 markstückgroßen, runden, steilgeränderten, schmutzig belegten, zum Teil konfluierten Geschwüren (ulceröse Syphilide), welche trotz der verschiedensten Behandlungsmethoden nur sehr geringe Tendenz zur Heilung hatten. Jetzt sind alle die Geschwüre unter Zurücklsssung runder, weißer, strahliger Narben geheilt. Wa s s erman positiv.

5. Pet e r P., 17 Jahre. Vater tot, Mutter lebt, angeblich gesund; Mutter S.-R. +. 6 Kinder leben; 7. Kind gleich nach Geburt gestorben. Peter ist 4. Kind. 1. Kind kann angeblich im Dunklen nicht sehen. 2. Kind gesund. 3. Kind hat kranke Augen und ist schwerhörig. 4. Kind ist Peter. 5. Kind hat kranke Augen. 6. Kind Anton (S.-R. + S. Nr. 6) z. Zt. in Augenklinik wegen Keratitis parenchymatosa. Als Kind völlig gesund; mit 6. Lebensjahr eine Zeitlang plötzlich blind; früher immer gesund. 
Mäßig, schlaffe Muskulatur. Schädel asymmetrisch. Seitenhöcker vorspringend. Linke Gesichtsseite wenig entwickelt. Nase wulstig, nicht sattelförmig. Radiare Narben auf der Oberlippe. Gaumen hoch. Zähne stark, oben teils doppelt stehend. Kubital- und Zervikaldrüsen geschwollen. Intelligenz gut. Augen: Keratitis parenchymatosa; bd. Zyklitis, die die Entfernung des rechten Auges wegen Phthisis bulbi nötig machte. S.-R. (Wassermann und Stern) positiv.

6. A $\mathrm{n}$ ton P., 14. Jahre. Bruder von Peter P. (S. N. 5). Vor einem Jahre ohne nachweisbare Ursache Kniegelenksentzündung rechts; seitdem kann er auch schlecht sehen, wovon vorher weder ihm noch seiner Mutter etwas aufgefallen war. Guter Ernäbrungszustand; Muskulatur kräftig. Innere Organe obne krankhaften Befund. Zähne rachitisch. Zervikaldrüsen erbsengroB, hart. Rechtes Knie: 30, 30, $27 \mathrm{~cm}$, Linkes Knie: 29, 30, $27 \mathrm{~cm}$. Bewegungshindernisse bestehen nicht. Augen: Keratitis parenchymatosa; keine Chorioretinitis peripherica. Wassermann positiv.

7. Franz H., 15 Jahre. Mutter angeblich augenkrank: Vater gesund. 1 Schwester $25 \mathrm{~J}$. und 1 Bruder $19 \mathrm{~J}$, gesund; Pat. ist 3. Kind. Vor 3 Wochen angeblich Sehstörungen entstanden $d u r c h$ Sand ins A u g e fli eg e n. MäBiger Ernährungszustand; Muskulatur schlaff. 1-58 groß; Brust 73-78, flach. Innere Organe o. B. Brustwirbelsäule nach rechts verbogen; rechte Schulter tiefer. Kleiner, asymmetrischer Schädel. Nase nach rechts verbogen. Radiare Narben auf den Lippen, vom Lippenrot auf Lippenweiß übergehend. Hutchins on s c he Zähne. Zervikaldrüsen und Inguinaldrüsen, erbsengroł. März d. J. Gelenkrheumatismns beider Kniegelenke; heilte in 6 Wochen ab auf Pulverbehandlung. Beide Kniegelenke geschwollen, besonders rechts. Rechts: $32,34,28 \mathrm{~cm}$; Links: $31 \cdot 5,32$, $28 \mathrm{~cm}$. Keine Ergüsse oder Bewegungshemmungen. Auf Röntgenbila normale Knochenenden. Auffallendes Erkalten (innerhalb weniger Minuten) beider Hände wie bei $\mathrm{R}$ a y n a ud. Keratitis parenchymatosa beiderseits. S.-R. +. Im Beginn der Schmierkur Schwellung sämtlicher Drüsen (Lokalreaktion). In einer exzidierten Drüse wird ohne Erfolg nach Spirochaeten gesucht. Hg-Kur keine wesentliche Besserung.

8. Anna H., 20 Jahre. Vater unbekannt; Mutter Krampfadern, sonst nibil (unehelich). Im 19. Lebensjahr sollen beide Kniegelenke nach häufigem Kuhmelken geschwollen sein; Sehbeschwerden, früher angeblich niemals, $\epsilon$ ntstanden durch Schlag eines Kuhschwanzes. Ziemlich gut genährt. Muskulatur schlaff. $20 \mathrm{Jahre;} \mathrm{1.61} \mathrm{Körpergröße.} \mathrm{Brust} \mathrm{75-79} \mathrm{cm}$, flach, besonders 1. Seite. Inneren Organe o. B. Lendenwirbelsäule etwas nach links gebogen. Breitschädel. Starke Asymmetrie des Gesichtes; nach links hinüber verschoben. Leichte Sattelnase; breit; weite offene Nasenlöcher. Hutchinson angedeutet; Zähne vollzählig, anscheinend sonst gesund, schließen nicht übereinander. Hörfähigkeit gut. Zervikaldrüsen erbsengroß, hart; desgleichen Inguinaldrüsen. Über der Lendenwirbelsäule 2 pfenniggroße, eingelegte, strahlenförmige Narben (Geschwüre als Kind, die auf Lebertran und Bäder zurückgegangen sein sollen). Normale Gelenke bis auf Kniegelenke. Die Konturen beider Gelenke verstrichen, besonders 
links. Links starke Schwellung im oberen Rezessus. Hier Kapselverdickung. Umfang: links: über Patella 38, Mitte 37, unter $31 \mathrm{~cm}$; rechts: über Patella 36.5 , Mitte 35.5 , unter $32.5 \mathrm{~cm}$. R. Unterschenkel (oberes Ende) verdickt; springt deutlich nach innen vor. Gehen macht Schmerzen; Beugen und Strecken vollständig. Augen: Keratitis parenchymatosa. Wa s sermann negativ.

9. Käthe M., 16 Jahre alt. Eltern leben; angeblich gesund. Eine Schwester lebend. 5 Geschwister zum Teil als ganz kleine Kinder gestorben; davon sind 2 älter gewesen als sie selbst. Mit 9 Jahren ziemlich plötzlich erblindet, innerhalb von 2 Monaten. Mäßig, Muskulatur schlaff. Sehr anämisch, Verdacht auf Tbk. Skoliose. Keine Säbelbeine. Schädel klein; Stirn niedrig, flach. Asynmetrisches Ge sicht. Radiäre Narben an den Lippen. Zähne gat und gesund. Ohren nichts. Keine Drüsenschwellungen. Haut trocken. Haare kurz. Intelligenz normal. Augen: Atrophia nervi optici. (An den Gefäßen weiße bindegewebige Stränge.) Seroreaktion + .

10. Elli P., 10 Jahre alt. Mutter Herzfebler; Vater angeblich gesund. 1 Bruder; 4 Jahre (epileptiforme Anfälle) keine tot. Elli ist 1. Kind. Vor einem halben Jahre traten die ersten Sehstörungen auf. Ziemlich guter Ernäbrungszustand, Muskulatur kräftig. Geringe Verbiegung der Brustwirbelsäule nach rechts. Inneren Organe ohne krankhaften Befund. Zervikaldrüsen erbsengroß. Haut braun. Keratitis parenchymatosa. Epileptiforme Anfälle (z. B. bei Blutentnahme) gehen sehr schnell vorüber. Wassermann positiv.

11. W e rner L., 11 Jahre; Bruder von Josef L. (s. Nr. 12). Im 6. Lebensjahre blieb die Sprache aus. Im 8. Jahre lernte er wieder sprechen. Im 9. Jabr wurde er im AnschluB an eine langsam sich entwickelnde Kehlkopfstenose tracheotomiert in chir. Klinik hier. Angeblich lag damals eine diphtherische Infektion vor. Als Kind von 2 Jahren Schwellung beider Ellenbogengelenke. Vor 2 Jahren nach Fall Bruch des Nasen beines. Vor $1 \frac{1}{2}$ Jahren augenkrank; an Ohren niemals etwas. Ernährungszustand schlecht; Muskulatur schlaff. Brust flach. Augen: Chorioretinitis atrophikans. Keratitis parenchymatosa. Alte Iritis. GroBer Schädel ; asymmetrisch. Lippen etwas fissuriert. Zähne schlecht, bröcklig, klein. Zervikald̈rüsen geschwollen. L. Kryptorrchismus. Geistig wenig zurück. Wassermann positiv.

12. J o s e L L., 14 Jabre alt. Mutter gibt luetische Infektion vor der Ehe zu. 23jähriger Bruder lebt, hört schlecht. 2. Mädchen starb im Alter von 6 Jahren; Ursache ? 3. Mädchen starb im Alter von 10 Tagen (Frühgeburt) an Lebenschwäche. 4. Totgeborene Frucht. 5. 16jähriger Sohn, gesund. 6. Abort im 3. Monat. 7. Ist Josef. 8. Werner 11 Jahre (S. Nr. 11). 9. Gesund. 10. Unentwickelt, Tbk. 11. Abort im 3. Monat. Seit Geburt in körperlicher und geistigerEntwicklung zurückgeblieben : lernte mit 3 Jahren laufen und sprechen. Im Alter von 10 Jahren zum 1. Mal Augenbeschwerden, schlechtes Sehen. Keratitis parenchymatosa. Masern, sonst nihil. Schlechter Ernährungszustand, Muskulatur sehr schwach. 
Brust flach, normale Größe. Schmaler, länglicher Schädel. Geringe, Asymmetrie. Keine Sattelnase. Lippen mit Radiärnarben bedeckt. Zähne bröcklig, schlecht, stehen weit auseinander, geben keinen VerschluB des Mundes. Ohren groß; Haare blond. Typische kleine harte Schwellung der Zervikaldrüsen, sehr viel Kubitaldrüsen. Auf Schleimbaut des harten Kiefers großje strahlenförmige Narbe nach der Mitte des hohen Gaumens hinziehend; soll niemals ein Ulkus dort gehabt haben. Augen: Abgelaufene Keratitis parenchymatosa; Chorioiditis; Ophthalmoplegia interna. Zurückgeblieben geistig; begreift schlecht; ist ungezogen zu Hause. Was sermann negativ.

13. Fried a, V. 13, Jahre, Eltern gesund. 3 Geschwister angeblich gesund. Im Alter von 8 Jahren trat A ugenerkrankung a uf. Muskulatur schlaff; guter Ernährungszustand. Brust flach, sonst nichts besonderes. Keine Zeichen von Rachitis keine Knochendeformitäten. Innere Organe o. B. Schädel klein; asymmetrisch. Sattelnase. Rhagaden auf dem Lippenrot. Zähne sehr schlecht, keine Hutchinson. Zervikaldrüsen ziemlich grob, hart. Narbe an r. Halsseite. Ohrläppchen angewachsen. Intelligenz gering. Augen : Keratitis parenchymatosa. Wa s s er m a n n negativ.

14. Anna S., 17 Jahre alt. I. Die Mutter gibt an, daß Anna aus erster Ehe ihres Mannes stammt. Aus iher eigenen Ehe: 1. Kind im Alter von $1 / 4 \mathrm{Jahr}$ gestorben. 2. Kind $9 \mathrm{Jahre}$, gesund. 3. Kind tot. 4. Kind ist Heinrich (s. Nr, 15). 5. Kind imbezill. 5 Jahre alt; 6. Kind gesund. 7. Kind gesund. Mutter nie krank gewesen; nur Blasenkatarrh; S. R. +. Mann ist in 2. Ehe mit ihr verheiratet; S. R. - ; seine erste Frau soll geschlechtskrank gewesen sein. A us erster Ehe: 3 Kinder lebend, 2 Kinder tot (Ursache unbekanut). 3 lebende Kinder nicht gesund, davon 2. hört schlecht. 1. (Anna) 2. Kind gesund (angebl. L.), 3. soll an Drüsen leiden; soll im 8. Lebensjahre an Lueserscheinungen (Geschwïre am After) geliten haben; schlechte Zähne, hört schlecht. Anna D. Im Alter von 2 Jahren trat Augenerkrankung auf; im Alter von 5 Jahren wurde d. 1. Auge enucleirt; mit 8 Jahren rechts Hornhautentzündung. Sonst nur Diphtherie. Klagen: Schmerzen im rechten Knie und Magenschmerzen. Guter Ernährungsstand; kräftige Muskulatur. 8. Geringe Asymmetrie des Gesichtes; Sattelnase. Zähne schlecht, keine Hutchinson. Lingua plicata. Narben auf den Schultern angebl. infolge von Furunkela im 15. Lebensjahre. Seit $1 / 2$ Jahr Schmerzen im rechten Knie, welches auch zeitweilig anschwellen soll. Ein ErguB ist nicht zu konstatieren, nur eine geringe Druckempfindliehkeit am r. Kondylus entlang des Unterschenkels. Menstruation regelmäßig. L. Auge enukleiert (angebl. infolge Keratitis). Wassermann positiv.

15. Hein rich D., 6 Jahre alt (s. Nr. 14). Seit 2 Jahren bemerkt die Mutter Schwellungen beider Liniegelenke und Eiterung beider Augen. Kleiner, aufgeweckter Knabe in gutem Ernährungszustand. Innere Organe o. B. Hydrozephalus mit starker Assymmetrie und typischer Sattelnase. Beide Kniegelenke erheblich geschwollen, so daß die Knie nicht völlig gestreckt werden können; keine Veränderungen der Knochen selbst 
Omfang oberhalb der Patelle: Links $23.5 \mathrm{~cm}$. Rechts $24 \mathrm{~cm}$. Beiderseitige Keratitis parenchymatosa; rechts außerdem eine Dakryozystitis. Zervikalund Inguinaldrüssen typisch geschwollen. Beroreaktion: positiv. Innunktionskuren und Syrup. ferri jodat. 10./XII. 09. Keine wesentliche Besserung; objektiver Befund derselbe. Wäbrend er anfangs in der Schule gute Fortschritte machte und früher auch recht aufgeweckt war, bleibt er jetzt erheblich hinter den anderen Kindern zurück. Durch mehrere $\mathrm{Hg}$-Kuren ist die Seroreaktion jetzt negativ.

16. Hans S., 20 Jahre. Eltern leben. 5 gesunde Geschwister. 1 tot. Mit 9 Jahren Sehstörungen. Guter Ernährungszustand; kräftige Muskulatur Starke Verbiegung der Wirbelsäule; sonst keine Knochendeformităt Hochstehende rechte Schulter. Herztätigkeit sehr beschleunigt. Ausgesprochener Hydrocephalus mit erheblicher Assymetrie; Unterkiefer sebr klein; vorspringend. Typische Cervikaldrüsenschwellung. Keine Mißbildungen. Augen: Keratitis parenchymatosa. Ohren groß. W as serman n peritis.

17. A uguste B., 9 J. Mutter soll an Asthma leideu Vater gesund. 1. Kind blind (13 Jabre). 2. Kind angebl. gesund (11 Jahre). 3. Kind ist Auguste. 4. Kind angebl. gesund (8 Jahre). Alle Kinder nach Bericht de: Augenklinik an Chorioretinitis erkrankt. \& Kinder (vor diesen und zwischen) als kleine Kinder gestorben; außerdem mehrere Aborte. Nov. 1908, also im Alter von 8 Jahren, an Chorioretinitis erkrankt. Muskulatur schlaff; guter Ernährungazustand. Inneren Organe ohne krankhaften Befund. Leichte Kyphose. Flachschädel. Gesicht assymetrisch. Ausgesprochene Sattelnase. Lippen mit tiefen radiären Narben. besonders Unterlippe. Zähne schlecht; Ohren gut. Haare, Zunge nichts. Augen: Chorioretinitis. Wassermann positiv.

18. Heinrich L., 50 Jahre. Ein Bruder im Alter von 25 Jahren lebt. Erstes Kind seiner Mutter eine Totgeburt. Zweites Kind ist er. Drittes Kind ist lebender Bruder. Viertes Kind ist Totgeburt. Fünftes Kind ist Totgeburt. Patient will als Kind sehr schwach gewesen sein; kann seit 2. Lebensjahre nicht gut sehen. Im Alter von 4 Jahren Uvula verloren. Großer Mensch, in gutem Ernährungszustand. Der Leib ist aufgetrieben, hart; eine Vergrößerung der Leber läßt sich nicht feststellen. Die Unterarme und Unterschenkel sind gegenüber den Oberarmen und Oberschenkeln kurz; die überhaupt schwache Muskulatur ist hier noch schwächer. Nase breit, eingefallen; Gesicht assymmetrisch. Gaumen hoch; Uvula fehlt; Narben auf der hinteren Pharynxwand. Hutchins on sche Zähne. Vorspringender Unterkiefer. Keine Drüsenschwellung. Über den ganzen Körper verbreiteter, seit 17 Jahren bestehender Lichen ruber planus. Wasser mann negativ.

19. Cla u 8 K., 13 J. 22./IX. 1908 bis 18./XII. 1908. Medizin. Klinik. Vater gibt luetische Infektion zu; ein Bruder ist gesund, Cl. erkrankte im Alter von 8 Jahren an einer Keratitis parenchymatosa. Seit ungefähr 6 Monaten klagte $\mathrm{K}$. über Schmerzen in der Magengegend; zeitweiliges Erbrechen; Stiche in 1 . Brustseite. Cl. soll von jeher viel an Schnupfen 
gelitten haben. Mittelgroßer schmächtiger Knabe von blasser Gesichtsfarbe. Tp. abens $38.5^{\circ}$. Leichte Kyphose; Brust flach. Abdomen: Sehr aufgetrieben; Umfang $75^{1} / 2 \mathrm{~cm}$. Man fühlt unter $r$. Rippenbogen große. runde, etwas höckerige, druckempfindliche Tumoren, anscheinend zur leber gehörig. Weiter unter fühlt man bei tiefem Eingehen keine Resitenz. Direkt in der Höhe des Nabels verlaufend fühlt man ganz hart und scharf den unteren Leberrand. Ganze Leber druckempfindlich. Leber $15: 16: 81 / 2$. Milz: 121/2 : 11 überragt Rippenbogen gut 2 Querfinger breit; sehr hart; etwas druckempfindlich. Stuhl und Appetit gut. Th or ax: schmal; Atmung symmetrisch aber wenig ausgiebig. Herz: o. B. Drü sen: Inguinal, Zervikal, Acheeldrüsen hart, klein, zahlreich. S. R. positiv Unter J. K. und Schmierkur iet Leberschwellung zurückgegangen; bei Entlassung Umfang des Abdomen $68 \mathrm{~cm}$. Diagnose: Hepatitis und Splenitis interstitialis luetica. 29./X. 1909. liegt wegen Verschlimmerung seiner Leber in der Mediz. Klinik. Linksseitige Hernie. Keinerlei Erscheinungen von seiten des Knochensystems, der Gelenke, der Haut und der Schleimhäute. Geistig völlig normal. August 10. Wegen zunehmender Leberschwellung mit 606 Ehrlich behandelt; ohne Erfolg.

20. M in n a D., 14. J. Mutter angeblich nie krank; hat negative S. R. Vater ebenfalls. 1. Kind im Alter von 8 Jahren gestorben an Pneumonie; soll gesund gewesen sein. 2. Kind ist 17 Jahre alt; völlig gesund. 3. Kind ist Minna. 4. Kind ist $13 \mathrm{Jahre}$; angeblich gesund. 5. Kind Zwillinge: davon 1. Kind im Alter von 8 Wochen gestorben; 2 Kind lebt und ist gesund; kann gut hören und sehen. 7. u. 8. Aborte (artifizielle). Großvater der Kinder soll Schiffskapitän gewesen sein; die Mutter von Minna soll mit Ausschlag geboren sein. Es leben von Mutter 6 Geschwister, 7. tot; die Lebenden sollen gesund sein; von den Toten 1 an Gebirnentzündung gestorben. In Alter von $9 \mathrm{~J}$ a hre n erkrankte Minna plötzlich am rechten Auge; lag damals hier in Augenklinik; vor 2 Jahren erkrankte auch das linke. Vor 1 Jabre trat in Anschluß an einen Fall Schwellung des r. Kniegelenkes, später des l. Kuies, seitdem tritt häufiger eine Schwellung. auf. Gut genährt; Muskulatur kräftig. Nichts Auffallendes, weder an Zähnen, noch Lippen usw. Geginge harte Schwellung der Zervikaldrüsen. Haut nihil. R. Kniegelenk geschwollen; Konturen verstrichen; keine Bewegungshinderung oder Schmerzhaftigkeit; Knochenstruktur normal. Augen Keratitis parenchymatosa.

21. Walter vo n F. Vater an Apoplexie gestorben; hat syphilitische Infektion gehabt. Mutter an Gehirnerweichung gestorben. 1. Kind an Diphterie gestorben. 2. gesund (völlig). 3. u. 4. tot geboren. 5. Kind ist Patient, hat erst mit 3 Jahren Laufen gelernt; war schwächlich. Mit 9 Jahren Geschwüre an den Füßen. Mit $8 \mathrm{Jahren} \mathrm{Augenentzündung.} \mathrm{Seitdem}$ fast dauerd in ärztlicher Behandlung; immer viel Geschwüre gehabt. Klein; Ernährungszustand gut; Muskulatur schlaff. An den inneren Orgauen nichts Auffallendes. Großer, oben flacher, sehr umfangreicher unten schmaler, asymmetrischerSchädel. Typische H u t c h in s on s c h e Zäbne Sehr hoher Gaumen, diffuse Alopezie. Geringe Schwellung der Zervikal- 
drüsen. Runde, oberflächliche Narben an Unterschenkeln. Augen Keratitis parenchymatosa bds. Chorioiditis. Wassermann positiv.

22. M a ri e R., 19. J. Vater 1861 syphilitisch infiziert; Mutter mebrere Male abortiert. 5 Kinder leben, 7 Kinder tot, im Alter von $1 / 2-8 / 4$ Jahren. Ältestes Kind gesund; 2. 1/2 Jahr alt gestorben; 3. ist Marie. Die nach Marie geborenen 3-4 Kinder gestorben; letzten Kinder ganz gesund. Marie war immer klein und schwächlich. Im Alter von $11 \mathrm{~J}$ ahren A ugenentzündung; allmählich zunehmende Schwerhörigkeit. Menses im 18. Lebensjahre. Wenig entwickeltes, schwächliches Mädchen. Schneidezähne: Hatchin s on. Zervikaldrüsen vergrößert. Lungen und Herz o. B. Knochenbau grazil. R. Tibia stark verdickt, höckrig; dsgl. 1. Tibia. J. K. Hg besser. Bds. Keratitis; Chorioditis; Taubheit: Zähne; Tibia e. S. R. wurde nicht gemacht.

23. Magdale ne F., $11 \mathrm{~J}$. Eltern angeblich nie intiziert. Geschwister gesund, 1. im Alter von $3 \frac{1}{2}$ Jahren an Diphtherie gest. $5 \mathrm{~J}$ ahre alt: dicke Kniegelenke. 7 Jahre alt: Scharlach und Nephritis; zugleich Schwellung der FuB-, Hand- und Ellenbogengelenke; Operation in chir. Klinik: 1. Tibia. Incision: Granulationshöhle mit Eiter. 9 Jahre alt; Entzündung des r. A ug e s und Löcher an beiden Gaumen; später: Entzündung des l. Auges. Großes, schwächliches Kind. Narben am Mundwinkel und über krista tibiae 1. Vorderfäche der Tibien abgerundet. Auf der Grenze zwischen harten und weichen Gaumen perforiertes erbsengrobes Ulkus. Kornea: links getrübt uud undurchsichtig; Papille normal. Chorioiditis aequatorialis mit kleinen pigmentierten Herden. Rhinitis; Nasengrund eingefallen. Uvula dünn. J. K. und Schmierkur; darunter heilt das Ulkus am Gaumen. S.-R. wurde nicht gemacht.

24. Anna S ch., 33 J. Vater soll viel krank gewesen sein; an Herzleiden gestorben; Mutter hat ein serpiginöses Syphilid im Nacken. 5 ältere Geschwister sollen gesund sein; 8 jüngere als kleine Kinder gestorben, im 14. Lebensjahre soll sie $1 / 2$ Jahr blind gewesen sein; seit jener Zeit datiert auch das Her zk o p f en. Mittelgroß, wenig kräftig, Muskulatur dürftig, schlaff, Sehleimbäute anämisch. Gewicht: $55^{1 / 2} \mathrm{~kg}$. Thorax mäßig gewölbt. Keine Zeichen von Rachitis oder Tuberkulose. Gesichtschädel schwächer ausgebildet als Gehirnschädel; Umfang $54 \mathrm{~cm}$, Nasenwurzel eingefallen (Sattelnase), Geringe Schwerhörigkeit. Zähne defekt. Pupillen beiderseits gleichweit, reagieren auf Lichteinfall gut. Bds. geringe Opticusatrophie, infolge abgelaufener Neuritis optica: Verwaschene Grenzen; Atrophie der Aderhaut in der nächsten Umgebung der Papille. Am r. Auge ganz temporal an der äubersten Peripherie große schwarze Pigmentklumpen; äquatoriale abgelaufene Chorioiditis. Lunge, Leber, Milz: nibil. Haut: Narben im Gesicht, wie Poukennarben. Herz: SpitzenstoB im V. J. c. R., $9 \mathrm{~cm}$ nach links von der Mittellinie, schwach fühlbar, daselbst starke Empfindlichkeit. I. Ton an der Spitze dumpf und paukend; über Aorta 1. Ton unrein; 2. Töne in der Basis gleichlaut. Deutliche Sternaldämpfung, Pulsation im Jugulum, Breite der Sternaldämpfung $8 \mathrm{~cm}$. Puls gespannt, sch wer zu unterdrücken, keine ausgesprochene Sklerose. Blutdruck am r. Ober- 
arm $215 m m$ Hg. Rö $n \mathrm{tg}$ e $\mathrm{n}$ bild: Deutliche Verbreiterung der Aorta, namentlich rechts von der Mittellinie ; Pulsation ungleichmäßig (offenbar sklerotisch). Von seiten des Nervensystems nichts besonderes. Häufig heftige Anfälle von Herzklopfen. Diagnose: Sklerose der Aorta. Wird wegen desselben Leidens noch zweimal in die Klinik aufgenommen (1907 und 1908); jedesmal keine wesentliche Besserung.

25. Emma B., 7 J. alt. Mutter Luesinfektion vor ungefähr 15 Jahren; hat nur ganz kurze Schmierkur gemacht; Vater nicht krank; geheiratet vor 8 Jahren. Vor der Heirat: 1. Kind gesund, lebt. 2. Kind Frühgeburt von 6 Monaten. 3. Kind Frühgeburt von 7 Monaten. Nach der Heirat; 4. Junge lebt, gesund, nur Drüsen; hört und siebt gut. 5. ist Emma. 6. Kind im Alter von $1^{1 / 2} \mathrm{Jahr}$ an Keuchhusten gestorben. Emma hat mit 3 Jahren Laufen gelernt; mit drei Jahren die ersten Sehstörungen. Keine Kniegelenksschwellungen: Muskulatur kräftig; Ernährungszustand gut. Viereckiger sehr flacher Schädel. Assymmetrie des Gesichtes gering. Nase: Typische Sattelnase. Lippenweich der Oberlippe tief fissuriert. Zähne schlecht, bröcklig; z. T, ausgefallen. Ohren groß Keratitis parenchymatosa. Zervikgldrüssen klein, hart. Wassermann positiv.

26. Minna H., 22. J, Eltern gesund; Vater angebl. Trinker. 5 Geschwister leben, alle völlig gesund; 4 Geschwister tot (3 gleich nach der Geburt; 1. 7 Wochen alt; Ursache unbekannt). Minna wurde nach den 4 toten geboren, ist also die älteste der lebenden Kinder. Ist wegen grober Schwäche mit 9 Jahren zur Schule gekommen; wurde seit ihrem 4. Lebensjahr wegen "Rachitis" dauernd behandelt; o hne jeden Erfolg; damals konnte sie wegen geschwollener Knie und FuBgelenke nicht gehen. Im 10. Lebensjahre bekam sie Schmerzen im r. Ellenbogengelenk; Arzt diagnostizierte Tbk. wurde wenig behandelt, im Laufe eines Jahres trat die jetzt bestehende Verkrümmung auf. Im Alter von 15 Jahren Entzündung des r. Kniegelenks (Rezidiv). Daraufhin blieb r. FuB im Wachstum zurück. Im Alter von 17 Jahren Entzündung des r. Schultergelenks; hier in chirurg. Klinik fünfmal Punktion. Seit vorigem Jahr Schwellung beider Handgelenke. Fühlt sich sehr matt, kann sich kaum selbst vom Stuhl erheben. Muskulatur schlaff; Appetit gut. Brust flach, besonders 1. Seite, 73-76 cm. Ganze 1. Körperseite in der Entwicklung zurückgeblieben. Geringe Skoliose. Verbiegung des l. Unterarmes; ungleich entwickelt. Verkürzung des 1. Unterschenkels; säbelscheidenförmige Verbiegung des l. Unterschenkels. R. Schultergelenk: Arm kann aktiv nur bis zur Horizontalen gehoben werden; passiv starker Widerstand, Knacken, Schmerzen; auch die seitlichen und Rückwärtsbewegungen erheblich beschränkt. R. Ellenbogengelenk: Nicht völlig zu strecken, wohl zu beugen. Erheblich verdickt; $21 \mathrm{~cm}$ gegen 19 links. L. Ellenbogengelenk: Ebenfalls nicht völlig zu strecken. Unterarmmuskulatur sehr atrophisch; an beiden Ellenbogengelenken Kapselschwellung. Hand ge lenke: Eine Handbreit oberhalb der Gelenke beginnt eine ziemlich plötzliche Schwollung der Gelenke. Schwellung in der Tiefe hart, ober- 
flächlich, prailelastisch, von der Krapsel herrührend. Beiderseits besteht eine völlig gleichmäßige Verbiegung nach außen. Finger: dick. Auffallende gleichmäßige Schwellung beiderseits aller 2. und 3. Metaphalangealgelenke; große Schwäche in den Händeu. R. Hüfte: Höher wie die linke. L. Oberschenkel: Weniger umfangreich wie der rechte. R. Knie: 34, 34, 30. L. Knie. 34, 34, 29. Die Konturen der Gelenke völlig verstrichen. Es fällt eine Verdickung mit starkem Knochenvorsprung des $r$. Oberschenkels oben auf; hier starke Verbiegung nach außen; sonst erscheinen die Knochenenden (Ober-, Untersch.) im Gelenk verdickt. R. Oberschenkel infolge der scharfen Verbiegung im oberen Drittel nur $5 \mathrm{~cm}$ kürzer. L. Untersehenkel nach vorn außen säbelscheidenförmig verbogen. Beide Fußgelenke Konturen verstrichen; keine Beschränkung der Bewegungsfähigkeit. Kleiner runder stark asymmetrische Schädel. Sattelnase; Lippen nihil; Ohren kiein, nichts Auffallendes. Zähne: schlecht, durcheinanderstehend. Haare: wenig. Zunge: nihil. Zahlreiche harte, dazwischen auch weiche Zervikaldrüsen. Haut: nichts, keine Narben. Hoher Ga umen. Narben: strahlige am Gaumen. Innere Organe gesund; seit 17. Lebensjahr regelmäßige Menses. Augen: nibil. Wassermann positiv.

5./II. 1910. Eine geringe Besserung ist im l. Handgelenk eingetreten; hier geringe Abnahme der Schwellung. Im 1. Schultergelenk nebmen die Schmerzen wieder zu. Auf der Röntgenplatte siebt man eine starke Auftreibung der Epiphysen der Unterarmknochen; die Knochenknorpelgrenze ist nnregelmäßig, die Knochenstruktion verwascheu. Dieselben Knochenveränderungen an der Tibia. Periostitis ossificans am 1. Radius im oberen Drittel.

27. 0 t to L., 5 J. Vater angeblich nie krank; S.-R. negativ. Mutter: S.-R. positiv, weiB angebl. nichts von einer luetischen Infektion. 2 Aborte; dann Otto geboren; dann Kind 2 Tage alt gestorben, dann Abort, dann Frühgeburt im 7. Monat, ist 2 Monate alt geworden; darauf Abort, darauf Frübgeburi im 5. Monat; jetzt gravida im 3. Monat. Ziemlich guter ¡Ernährungszustand. Linke Schulter etwas tiefer. Schiefschädel. Asymmetrie des Gesichtes. Sattelnase. Lippen mit radiären Narben bedeckt. Schueidezähne ausgesprochen Hutchinson. Inneren Organe o. B. Zervikaldrüsen erbsengroß. Vor einem Jahre schwollen beide Kniegelenke, nachdem kurz vorher sich schlechtes Sehen bemerkbar gemacht batte (Keratitis parenchymatosa). Nach Schmierkur trat die Schwellung der Knie auf, die nicht zuräckging. Prof. Noesske Chir. Klin. Punktion: seröse Flüssigkeit. Links: 22, 24, 19.5, rechts : 22, 24, 20. Eine Schmierkar hier brachte lkeine Besserung; seitdem wieder Verschlimmerung der Augen. Beine können nicht völlig gestreckt werden. Punktion des r. Kniegelenks; darin ziemlich viel seröse Flüssigkeit. Augen : keratitis parenchymatosa S.-R. (W. u. B.) potitiv. S.-R. mit der serösen Punktionsflüssigkeit aus r. Kniegelenk positiv. $\mathrm{Nach} \mathrm{Hg}$ ist keine Besserung eingetreten; doch ist auch der Prozeß an den Augen durch größere Mengen von Jodeisen zum Stillstand gekommen. 
28. Heinrich Sch., 24 J., unehelich geboren. Von seiner Mutter und seinem Stiefvater 2 Kinder; davon 1. im Alter von 2 Monaten (er war 6 Jahre alt), das zweite im Alter von 3 Wochen (er war 16 Jahre alt) gestorben. Ursache unbekannt. Mit $11^{1 / /} J$ ahren bds. Kniegelenksschwellung und Schwäche; Puntion; Heilung; 14 Tage nach Entlassung rote Augen; Sehvermögen schlechter; seitdem Augenbeschwerden; hänfiger Rheumatismus. Mäßiger Ernährungszustand; Muskulatur nicht sebr kräftig. Brust flach. Inneren Organe o. B. Langschädel. Schlechte Zähne. Seit $2^{1} / 2$ Jahren verbeiratet; 2 gesunde Kinder. Alte Keratitis parenchymatosa bds., rechts mit Glaukom, links mit chronischer Iritis. Wassermann positiv.

29. Otto G., $13 \mathrm{~J}$. Otto ist erster Sohn in der Ehe; keine Aborte vorher; Mutter und Vater angeblich gesund. Mutter S.-R. +, Vater S.-R. - . 2. Kind im Alter von 9 Wochen gestorben; Ursache unbekannt. 3. Kind gesund. 4. Kind augenkrank. 5. Abort im zweiten Monat. Im Alter von 5 Jahren Masern. April ds. Js. Schwellung beider Kniegelenke; seit 4 Wochen kann er schlecht sehen. Fraktur des rechten Armes. Mäßiger Ernährungszustand; Muskulatur schlaff. Körpergröße 1.46; Brust 69-74.5. Geringe Lordose. Säbelscheidenförmige Verbiegung der 1. Tibia Sonst keine auffallendeu Veränderungen außer schlecht verheilter Fraktur. Flacbschädel; Asymmetrie des Gesichts. Sattelnase. Unterkiefer weit vorstehend. Zähne: Sehr schöne Hutchinson, auch der unteren Reihe; klein, aber sonst gesund aussehend. Ohren abstehend; Gehör gesund. Haare und Zunge nihil. Augen: Keratitis parenchymatosa links. Zervikaldrüsen erbsengroß. Weiche glatte Haut, keine Narben. Beide Knie gleichmäßig stark geschwollen, besonders in Gegend der Kondylen. Kapselschwellung. Röntgenbild: Normale Knochen. Wassermann positiv. 1./II. 1910. Unter energischer $\mathrm{Hg}$-Behandlung ist Besserung der Augen eingetreten, so daß V. jetzt ziemlich gut sieht.

30. Daniel V., 12 J. Vater negiert Lues, konzediert Go. 1. Kind totgeboren. 2. Kind: Daniel. 3. u. 4. Kind gesund (können gut hören und seben). Mit 3 Jabren Masern; mit 4 Jahren Haarausfall. Im Alter von $10 \mathrm{Jahren} \mathrm{Schwellung}$ beider Knie. Augenstörungen seit 6 Wochen. Ernährungszustand gut; Muskulatur schlaff. $147 \mathrm{~cm}$ groß; Brust 67-71. Inneren Organe o. B. Leichte Lordose der Wirbelsäule. Außer Säbelscheidentibien nichts am Knochensystem. Schädel rund; geringe Asymmetrie; hoher Gaumen. Runder, kleiner Schädel. Totale Alopezie. Augen: Keratitis parenchymatosa links. Zervikaldrüsen geschwollen. Knie: Nichts nachweisbar durch klinische Untersuchung. Wassermann positiv. Aug. 10. Wegen Verschlimmerung des linken Auges wird eine Injektion mit Ehrlich 606 gemacht. Das linke Auge bessert sich vorübergehend; aber zugleich tritt auch rechts eine Keratitis parenchymatosa auf.

31. E m m a K., 15 J. Vater lebt, gesund; hat vor 16 Jahren sich luetisch infiziert; schlecht behandelt; ein Jahr darauf geheiratet. 1. Kind im 8. Monat Frühgeburt, 2. Abort; 3. ist Emma. Erkrankte im Alter von 5 Jahren an einer ausgedehnten Chorioretinitis beiderseits. Musku- 
latur mäßig; guter Ernährungszustand. $15 \mathrm{~J} .1 .48$ groß; Brust flach; Mammae entwickeln sich. 1903 Hydrops genu bds. Jetat keine Kniegelenksschwellung; aber geringe Rauhigkeit beider Tibien. Röntgenbild zeigt normale Knochen in den Gelenken. Schiefschädel; keine offene Suturen. Strahlenförmige Narben am harten Gaumen. Inneren Organe o. B. Asymmetrischer Schädel. Unterkiefer vorspringend. Ausgesprochene Sattelnase (Ozaena). Hutchinsonzähne. Mitte des 1. Gaumens am Übergang zum weichen Gaumen ein jetzt 2 markstückgroßes rundliches Loch, welches vor etwa 3 Wochen in die Länge gezogen war mit ziemlich unregelmäßigen zackigen Rändern, die jetzt, nach 3 Wochen J. K., glatt sind. Pat. hat dieses Loch seit Mai vorigen Jahres bemerkt; wurde daraufhin in hiesiger Nasenklinik mit J. K. behandelt; Stillstand. Seit Mai d. J. langsame Vergrößerung der Perforation, weshalb in die Klinik. Halsdrüse links ziemlich stark geschwollen, hart. Menses im März d. J. zum erstenmal; seither nicht wieder. S.-R. positiv. Augen: Chorioretinitis diffusa, besouders an der Peripherie unten; Strabismus divergens; alte Hornhanttrübungen mit alten tiefen Gefüßen (Keratitis parenchymatosa). Es wird eine Hg-Injektionskur gemacht. Das Ulkus zieht sich zusammen; Wassermann wird negativ. 28. Mai 1910. Wiederaufnahme in die Klinik: Fast der ganze rechte $M$. stenocleidomastoides ist hart; geschwollen; vereinzelte kleine Ulzerationen; typische Muskelgummen. Wassermann positiv. Bis zu $3 \mathrm{mg}$ T. A. weder Allgemein, noch Lokalisation. Injektion mit Ehrlich 606 intraglutaeal $0.3 \mathrm{Gramm}$. Es tritt eine langsame, sich aber doch innerhalb 4 Wochen vollziehende Heilung ein. 2. Nov. 1910. Pat. ist jetzt völlig frei von Erscheinungen. Wassermann negativ.

32. Frau Henriette R. 30 Jahre. Mehrere ältere Geschwister sind tot; es leben außer ihr noch 2, die angeblich gesund sind. Pat. hatte 7 Kinder (4 gestorben, Brechdurchfall, Diphtherie, Krämpfe), 3 lebende Kinder gesund. Im Alter von $16 \mathrm{~J}$ a hren Augenentzündung rechts, von 20 Jahren links. Kleine Frau in mäßig gutem Ernährungszustand. Muskulatur schlaff. Geringe Asymmetrie des Gesichts. Zähne klein, weit auseinanderstehend. Keine Drüsenschwellungen; radiäre Narben an der Oberlippe. Innere Organe o. B. Augen: Keratitis parenchymatosa bds. Nach einer Schmierkur und J. K. Besserung. S.-R. positiv.

33. Melitta W. 19. Jahre. Mutter gesund; keine Erscheinungen einer alten Lues; negative S.-R.; Vater tot. Frstes Kind tot geboren. 2. Kind ist 30 Jahre; ebenfalls augenkrank. 3. Kind ist gesund. 4. Kind ist Melitta. Im Alter von 12 Jahren augenkrank; schnell geheilt. Vor 2 Jahren erkrankte sie erst auf dem linken, dann nach wenigen Monaten auf dem rechten Auge bis zur völligen Blindheit. Zugleich hatte sie heftie Schmerzen in beiden Knien. Ursache hierfür unbekannt. Mittelgroßes Mădchen in gutem Ernährungszustand. Muskulatur schlaff. Innere Organe o. B. Asymmetrisches Gesicht. Hatchinsonsche Zähne, die weit auseinanderstehen. Augen: R. Ange: Alte tiefe HornhautgefäBe; alte Synechien; Opticus; Peripherie o. B. L. Auge: Dichte tiefe Makulae 
mit alten Gefäßen, Präzipitatresten, alten Synechien. Vur einem Jahre auf Tuberkulinprobe positiv mit allgemeiner Reaktion. Auf Tuberkulin. kur Besserung des Auges, so daß sie ziemlich gut sehen kann. Wassermann positiv. (Kombination von Lues und Tbk.?)

34. Hedwig J. 31. Jahre. Mutter wegen spinaler Lues in Psych. Klinik behandelt. Zwei Schwestern angeblich gesund. Sie selbst hat drei Kinder, 11, 5 und 2 Jabre, gesund; eins tot an Schlaganfall. Im Alter von 26 Jahren zuerst augenkrank; vorher immer gesund. Kräftig gebaute Frau; Ernährungszustand gut. Innere Organe o. B. Keratitis parenchymatosa. Seroreaktion positiv. Ausgesprochene Melancholie.

35. Walter. B.. 12 Jahre. Mutter lebt vom Vater getrennt, der vor langen Jahren einmal Ausschlag (?) gehabt haben soll. Mutter hat negative Seroreaktion. Ein 10jähriges Mädchen ist bisher völlig gesund. Im 11. Jabr Schmerzen in beiden Kniegelenken; bald nachher Augenerkrankung. Schlank gebauter Knabe in gutem Ernährungszustand. Keine Deformitäten des Knochensystems. Beide Kniegelenke erscheinen gescbwollen; auf Röntgenplatte zeigen sich normale Knochen; keine Bewegungshemmung darin. Asymmetrischer Schädel mit vorspringendem Unterkiefer. Hutchinsonsche Zähne. Keratitis parenchymatosa. Intelligenz gut. Seroreaktion positiv.

36. Gustav H., $20 \mathrm{~J}$. Mutter lebt, gesund. Vater soll an einer Lungenkrankheit gestorben sein. 2 Geschwister sind gesund. Pat. will niemals krank gewesen sein. Anfang August erkrankte plötzlich sein linkes Auge. Ungefähr 14 Tage später schwoll ohne besondere Veranlassung das linke Kniegelenk an. Grober, schlanker Mensch in mäBigem Ernährungszustand. Das linke Kniegelenk ist um mehrere Zentimeter geschwollen; Bewegungen beschränt und schmerzhaft; keine akuten Entzündungserseheinungen darin. Keine Deformitäten von seiten des Knochensystems. Achsel-, Leisten- und Zervikaldrüsen geschwollen. Keratitis parenchymatosa links. Wassermann positiv. Das linke Kniegelenk wird punktiert und $45 \mathrm{ccm}$ einer serösen, leicht getrübten Flüssigkeit abgelassen. Wsssermann mit dieser Punktionsflüssigkeit eberffalls positiv. Innere Organe ohne krankhaften Befund.

37. Gustav Sch., 15 Jahre. Vater gibt mit Bestimmtheit an, daß weder er noch die Mutter luetisch gewesen seien. 1. Gravidität - Abort im 6. Monat. 2. Gravidität - Gustav; außerdem nooh 5 gesunde, lebende Kinder. G. wurde als gesundes und normal aussehendes Kind geboren und entwickelte sich bis zur Mitte des ersten Lebensjahres recht gut; von dieser Zeit an nahm der Schädel an Umfang unverhältnismäbig schnell zu und bildete sich zu einem Hydrozephalus herans. Im Alter von 6 Jabren beiderseitige Kniegelenksentzündung. Im Alter von 7 Jahren Augenentzündung. Seit einem halben Jahre schwillt die Nase an; zugleich tritt ein Geschwür auf dem Gaumen auf. Schlank gebauter Knabe; Muskulatur schlaff. Hydrozephalus; circumferentia fronto - occipitalis $57 \mathrm{Ztm}$. Abstand $z$ wischen Porus acusticus - Scheitelporus acusticus $39 \mathrm{Ztm}$. Sattelnase; es entleert sich ein schmierig eitriges Sekret aus der 
Nase. Weicher Gaumen und Uvula fast völlig zerstört. Gute Zähne. Alopecia diffuse. Augen: Beiderseits alte parenchymatöse Keratitis, Myopie und periphere Chorioretinitis. Kniegelenke o. B. (auch im Röntgen. bilde). Wassermann positiv. Auf Injektion von Ehrlich 606 tritt geringe Besserung des Ulkus am Gaumen ein.

An der Hand dieser Krankheiten möchte ich die wesentlichsten Frgebnisse im folgenden zusammenfassen.

Wohl bei keiner Krankheit läßt uns aus leicht begreiflichen Gründen die Anamnese so oft im Stich wie bei der Lues. 19 mal hat sie bei unseren Fällen völlig versagt; die Eltern gaben weder über ihre frühere syphilitische Infektion etwas an, noch brachte uns bei ihnen die objektive Untersuchung oder die Seroreaktion Aufschlüsse. 4 mal gaben die Väter eine frühere luetische Infektion zu, wollten aber von einer Übertragung auf ihre Frau nichts wissen. 19 mal wechselten gesunde und kongenital syphilitische Kinder miteinander ab. 8 mal konnte man wegen vorhergegangener oder nachfolgender Aborte und Totgeburten eine syphilitische Erkrankung der Mütter vermuten; 6 mal wußten die Mütter ron einer syphilitischen Infektion. 8 mal erhielten wir bei der serologis chen Untersuchung der Mütter eine positive Reaktion. Ich möchte hervorheben, daß auch diese letzteren Mütter klinisch völlig frei von irgendwelchen Symptomen waren. Wir finden also auch hier wieder die Bestätigung der alten Erfahrung, daß wir uns auf die Anamnese und den klinischen Befund bei der Mutter nicht verlassen dürfen, sobald der Verdacht auf kongenitale Syphilis des Kindes besteht. Am meisten hilft uns hier noch die Seroreaktion, wenn sie auch in keineswegs allen Fällen die gewünschte Aufklärung bringt. Wir haben ferner 9 ältere und jüngere Geschwister der oben angeführten Kranken zu untersuchen Gelegenheit gehabt, haben aber bei ihnen weder Zeichen einer ausgeheilten noch einer bestchenden Syphilis finden können.

Nach unseren Fällen berechnet sich der Beginn der Erkrankung durchschnittlich auf das 8. Lebensjahr. Die frühesten Erscheinungen der Syphilis wurden im 1. Jahre beobachtet; der älteste der mir zur Verfügung stehenden Fälle erkrankte angeblich im 26. Lebensjahre (Fall 34). In der Literatur sind allerdings Fälle beschrieben worden, in denen die ersten Erscheinungen noch in sehr viel späterem Lebensalter 
aufgetreten sind. Im Gegensatz dazu konnten wir bei 26 Fällen, bei denen luetische Erscheinungen erst sehr viel später beobachtet wurden, durch das Vorhandensein radiärer Narben oder auch $\mathrm{Hu}$ t c hin s o n scher Zähne den Schluß ziehen, daß fr ü h er syphilitische Symptome bestanden hatten.

Bei 4 Fällen waren wir nicht in der Lage, die Seroreaktion rorzunehmen. Von den übrigen reagierten 27 positiv; also wir fanden den hohen Prozentsatz von $73 \%$ positiver Reaktionen. tome?

Welches sind die häufigsten Krankbeitssymp-

Um hierüber ein Urteil abgeben zu können, muß ich zunächst in Kürze an der Hand unserer Fälle auf die einzelnen Krankheitserscheinungen, welche den meisten Fällen eigen sind, eing ehen.

16 mal haben wir ein oder doppelseitige Kniegelenkserkrankung beobachtet, darunter auch in 2 Fällen (Nr. 23 und 26) eine Mitbeteiligung anderer Gelenke, $19 \mathrm{mal}$ kamen Drüsenschwellungen vor, 26 mal Keratitis parenchymatosa und $26 \mathrm{mal} \mathrm{Knochendeformitäten}$ verschiedener Art.

Über die Erkrankung der Kniegelenke liegt eine eingehende Arbeit von Bos s e (Beiträge zur klinischen Chirurgie 1905/06, Bd. LI) vor. Er hat in 3 Fällen aus der Gelenkkapsel Probeexzisionen zur histologischen Untersuchung gemacht und fand dabei zahlreiches Granulationsgewebe mit mehr oder minder großem Gefäßreichtum und kleinzellige Infiltration. Radiologisch bat er eine keulenförmige Verdickung der oberen Diaphysenenden durch Periostitis ossificans und eine unregelmäßige Verkalkungszone der Epiphyse gefunden. Er hält das kliwische Bild der kongenitalen Gelenklues für abgeschlossen.

Auf Grund unserer Untersuchungen können wir seine Anschauung nicht ganz teilen. Nur einmal zwar konnten wir die Gelenkkapsel histologisch untersuchen. Das sich bietende mikroskopische Bild war ein so wenig typisches, daß man hieraus irgendwelche Schlüsse nicht ziehen konnte. Vor allem vermißten wir jegliche für Lues charakteristische Gefäßreränderung. In zahlreichen Schnitten konnten Spirochaete pallidae nicht ge- 
funden werden. Und doch sitzen nach unseren Beobachtungen die Veränderungen hauptsächlich an der Gelenkkapsel.

Von den 16 Fällen haben wir 10 Fälle rön tge nolog is ch untersucht, auch solche Gelenke, in denen sich akute Prozesse abspielten, wo also Gelenkergüsse vorhanden waren. Aber wir haben 9 mal an den Knochen selbst keine Veränderungen finden können. Diese Gelenke ließen sich nicht unterscheiden von gleichaltrigen gesunden Gelenken, die wir zur Sicherheit mitphotographierten. Aus diesem Grunde halten wir eine Beteiligung der Knochen selbst nicht für erforderlich, wenn wir auch vereinzelte, aber wenig charakteristische Rauhigkeiten an den Knochenenden beobachteten. Auch die sich klinisch häufig darbietende Verdickung der Knochenenden ist nur eine scheinbare und auf eine sehr derbe Verdickung der Gelenkkapsel zurückzuführen.

In zwei Fällen stellten wir mit der Punktionsflüssigkeit eines geschwollenen Gelenks (Fall 27 u. 36) eine Wassermannsche Reaktion an und erhielten einen positiven Ausfall.

Etwas näher möchte ich auf Fall 26 eingehen. Hier haben wir neben ganz erheblichen Verbiegungen und Verkrii mmungen der Unterarmknochen mit stellenweise vorhandener Periostitis eine starke Kapselveränderung zu verzeichnen zugleich mit ausgesprochenen Fipiphysenveränderungen besonders in beiden Handgelenken. Die Epiphysen sind verbogen und aufgetrieben und ihre Struktur zeigt ein unregelmäßiges Maschennetz. Die Kapsel ist geschwollen, weich und elastisch. In diesem Falle handelt es sich um starke Beteiligung mehrerer Gelenke. Leider konnten wir in einem zweiten Falle mit Beteiligung der Hand-, Fuß-, Knie- und Ellenbogengelenke (23) keine Röntgenogramme aufnehmen.

Klinisch ist noch hervorzuheben, daß die Kniegelenkserkrankung fast ausschließlich eine doppelseitige ist. Sie ist in 11 Fällen als e r s te s Symptom der sog. L. h. tarda aufgetreten. Differentialdiagnostisch kommt hauptsächlich $\mathrm{Tu}$ b e r$\mathrm{kulose}$ in Betracht. Die Kniegelenkstuberkulose hat aber nicht einen so akuten oder subakuten Charakter, wie die Lues, 
die meist innerhalb weniger Wochen zu einem ausgesprochenen Krankheitsbilde führt. Die Tuberkulose kann primär sowohl in der Kapsel wie im Knochen ihren Anfang nehmen; während sich die Lues bei der sog. Hereditaria tarda wenigstens nach unseren Frfahrungen in der Mebrzahl der Fälle in der Kapsel lokalisiert. Lassen sich aber dennoch im Röntgenbilde auch Knochenveränderungen nachweisen (Fall 26), so spricht ein positiver Wassermann mit großer Wahrscheinlichkeit für den luetischen Charakter der Erkrankung. Bei demselben Falle von Knochenlues haben wir auch $\mathrm{Tu}$ berkulininjektionen gemacht. Die Patientin reagierte auf hohe Dosen nicht, weder allgemein noch lokal; eine Pirquetsche Kutanreaktion fiel ebenfalls negativ aus, so daß man deshalb wohl berechtigt ist, mit Sicherheit eine Tuberkulose auszuschließen. Vor allem darf man sich aber nicht auf das weitere Hilfsmittel einer mehr oder minder erfolgreichen Therapie verlassen. Wie ich später noch hervorheben werde, leistet sie keineswegs etwas Zufriedenstellendes.

In 19 Fällen konnten wir deutliche Drüsenschwell ung en nachweisen. Meistens sind die Zervikaldrüsen erkrankt, weniger die Kubital- und Inguinaldrüsen. Sie unterscheiden sich in bezug auf ihre Konsistenz nicht von denen bei der akquirierten Lues; sie sind durchweg erbsengrob und hart. In Fall 7 beobachteten wir ein eigentümliches Phänomen. Als der Kranke einige Tage graue Salbe eingerieben hatte, bekam er ein schwaches Hg-Exanthem; zugleich hiermit trat eine deutliche empfindliche Schwellung sämtlicher Drüsen, besonders der Zervikal- und Inguinaldrüsen auf, die wieder zurückging, als das $\mathrm{Hg}$ fortgelassen wurde. Eine exzillierte Drüse wurde nach Levaditi auf Spirochaeten untersucht. Trotzdem eine sehr große Anzahl Schnitte sorgfältig durchsucht wurden, konnten wir nicht eine einzige finden. Es ist ja anzunehmen, dab den Lymphdrüsen auch bei der sog. L. b. tarda als Lagerstätte für die Spirochaeten eine gewisse Rolle zukommt; soviel ist aber wohl zu sagen, daß sie nur sehr spärlich in ihnen vorhanden sein. werden.

In 27 Fällen lag eine Keratitis parenchymatosa vor, zum Teil in Kombination mit Chorioiditis. Sie ist in 
der Hälfte aller Fälle (andere Ursachen z. B. Tuberkulose) auf erbliche Syphilis zurückzuführen; nur in $3 \%$ kommt die erworbene Lues in Frage. Sie verläuft bei der hereditären Lues verhältnismäßig stürmisch innerhalb weniger oder mehrerer Wochen und ist meistens doppelseitig, indem sie häufig gleichzeitig, noch häufiger aber erst das eine und dann das andere Auge befällt.

Kn ochendef ormitäten wurden 26 mal beobachtet bei unseren Fällen. Hierzu habe ich alle Veränderungen am Knochensystem gerechnet, die verschiedenen Formationen des Schädels, die dann oft eine ganz erhebliche Assymmetrie des Gesichts zur Folge haben. Die auffälligste Knochenveränderung, die Sattelnase, beobachteten wir 14 mal. Verhältnismäßig häufig treten Veränderungen des Skeletts an den Unterarmknochen, noch mehr aber an den Tibien auf. Letztere ist eine geradezu klassische Lokalisation der Lues hereditaria. Es handelt sich um eine wirkliche Verdickung und auch Verlängerung des Knochens. Stadler (Fortschritte a. d. Gebiete der Röntgenstrahlen 1907-08, Bd. XI) hat gezeigt, daß die verkrümmten Tibien ihre normale Struktur verloren baben, daß die feinen Maschennetze der Spongiosa verwischt sind; Spongiosa und Kompakta gehen ohne Grenze in einander über. Auch auf unseren Röntgenbildern finden wir diese Untersuchungsresultate bestätigt.

Von weiteren für L. h. sprechenden Erscheinungen ist die Taubheit noch zu erwähnen, die wir auffallender Weise nur bei 2 Fällen fanden. Sie hat ihre Ursache in einer Labyrintherkrankung, die schon innerhalb weniger Wochen, oft aber auch erst nach 1-2 Jahren zur völligen Taubheit führt.

Hutchins on sche Zähne konnten wir in 11 Fällen nachweisen; häufiger dagegen fallen die weit auseinander stehenden kleinen, unregelmäßig angeordneten Zähne auf. Man muß sich wohl hüten, die sehr oft vorkommenden rachitischen Zähne mit hereditär-syphilitischen zu verwechseln.

Besonders charakteristisch für Lues congenita sind die radiären Narben an den Lippen; wir haben sie in 8 Fällen zu verzeichnen. Sie sind offenbar Überbleibsel papulöser Effloreszenzen, die ulzeriert und in dieser Weise zur Heilung ge- 
kommen sind. Sie sind in ihrem Zuge so typisch, dab sie zu Verwechslungen keine Veranlassung geben können.

Nach unseren Erfahrungen gehören Narben auf der Haut, wie sie besonders Fournier hervorhebt, doch zu den Seltenheiten.

Auffallend ist bei unseren Fällen, daß wir nur 3 mal eine Störung der Intelligenz beobachteten, davon einmal noch eine ausgesprochene Melancholie. Allerdings fällt bei den Kindern, bei denen die parenchymatöse Keratitis zur völligen Erblindung geführt hat, eine einseitige Begabung auf, die aber wohl z. T. auf die Erziehung zurückzuführen ist, welche die vorhandenen Talente in besonderer Weise auszubilden sucht.

Welche Symptome beobachteten wir nun am häufigsten nebeneinander an demselben Fall?

1. Keratitis + Drüsenschwellung 1 mal (S. R. +).

2. Keratitis + Drüsenschwellung + Kniegelenksschwellung 9 mal (S. R. 7 mal +, 2 mal - ).

3. Keratitis + Kniegelenksschwellung $3 \mathrm{mal}$ (S.R. $3 \mathrm{mal}+$ ).

4. Knochendeformitäten + Keratitis 6 mal (S. R. $4 \mathrm{mal}+$, 2 mal -).

5. Knochendeformitäten + Keratitis + Drüsen $7 \mathrm{mal}$ (S. R. $5 \mathrm{mal}+, 2 \mathrm{mal}-$ ).

6. Knochendeformitäten + Keratitis + Drüsen + Kniegelenksschwellung $11 \mathrm{mal}$ (S. R. $10 \mathrm{mal}+, 1 \mathrm{mal}-$ ).

Nach dieser Zusammenstellung finden wir also in der Überzahl der Fälle Knochendeformitäten im Verein mit Keratitis parenchymatosa und Kniegelenksschwellungen. Neben der häufigen Keratitis und den Kniegelenksschwellungen, zwei sehr charakteristischen Symptomen, fällt aber auch die häufige Beteiligung der Drüsen auf.

Dab die kongenitale vis zerale Lues so selten beobachtet wird, hat seine Ursache darin, daß falls es wirklich zu einer 
Infektion der inneren Organe kommt, die schweren Störungen sehr früh zum Tode des Kindes führen müssen. (Nur bei Fall 19 eine Hepatitis interstitialis; und bei Fall 24 eine Aortitis.)

In welchem Verhältnis steht der Ausfall der Seroreaktion zu den Symptomen?

Aus der Zusammenstellung geht herror, daB die S eroreaktion dort am häufigsten positiv ausfällt, wowir die meisten Symptome haben. Welches der einzelnen Symptome am stärksten den Ausfall der Reaktion beeinflußt, ist nicht zu sagen. Sie $k a n n$ auch dann noch negativ ausfallen, wenn ausgedehnte Symptome vorhanden sind. Der hohe Prozentsatz des positiven Ausfalles $73 \%$ ist jedoch hervozuheben. Wir vertreten den Standpunkt, daß der positive Ausfall der Seroreaktion an aktive Spirochaetenherde gebunden ist. Auch die Lues congenita spricht nicht gegen diese Anschauung; denn wir sehen ebenfalls bei ihr, wenn auch selten, akute Exazerbationen und wir vermögen durch die Behandlung den positiven Ausfall in einen negativen umzuwandeln. Aber sehr viel schwerer, wie bei der akquirierten Lues; in genau demselben Maße sind auch die Symptome der hereditären Lues sehr viel schwerer zu beeinflussen.

Wie haben wir uns nun diese eigentümlichen Fälle zu erklären, in denen die Lues so lange latent bleibt, um dann plötzlich mit tertiären Symptomen aufzuflackern?

Pasini hat bei kongenital-luetischen Kindern die Spirochaeten in den Zahnkeimen nachgewiesen und damit die Ursache der $\mathrm{Hutch}$ ins on schen Zähne festgestellt.

Igershe imer (Deutsche med. Wochenschr. Nr. 20, 1910) hat in der parenchymatös erkrankten Hornhaut eines 14jährigen an Syphilis hereditaria leidenden Menschen eine echte Spirochaete pallida gefunden.

Nach diesen beiden Untersuchungsresultaten ist der wirklich syphilitische an die Anwesenheit der Spirochaeten gebundene $\mathrm{Ch}$ arak t er dieser beiden Symptome nicht in Zweifel zu ziehen. Wir müssen daraus schließen, daß überall dort, wo sich syphilitische Prozesse abspielen, auch Spirochaten vorhanden sind. 
Es besteht nun die Möglichkeit, daß nur ganz vereinzelte Spirochaeten von dem mütterlichen Organismus auf den kindlichen übergehen. Hiermit können wir vielleicht einige Formen der sog. tardiven Syphilis erklären, nämlich solche Fälle, wo wir langsam sich herausbildende, chronische Prozesse ror uns haben, wie z. B. bei Knochenerkrankungen. Entweder die Spirochaeten sind sehr frühzeitig bei der Anlage in die betreffenden Keime der Knochen hineingelangt und setzen hier Veränderungen, die zu den Entwicklungshemmungen und Mißbildungen fibren, in derselben Weise wie bei den $\mathrm{Hutch}$ in s on schen Zähnen. Oder aber die Spirochaeten gelangen erst nach der Geburt von irgendeinem im Körper vorhandenen Depot in die Knochen und setzen sich fest. Infolge ungünstiger Ernährungsbedingungen kommt es nicht zu einer Vermehrung der Spirochaeten und zur allgemeinen Infektion; der Prozeß bleibt auf ein Organ beschränkt. Hier führt dann die Anwesenheit der Spirochaeten zu einem Krankheitsprozeß, der langsam in Monaten oder Jahren die verschiedenen Stadien durchläuft, je nach dem Sitz, und uns erst zu Gesicht kommt, wenn bereits schwere destruierende Veränderungen aufgetreten sind. Wir sehen dann Knochenveränderungen, die von tertiär syphilitischen nicht zu unterscheiden sind.

Wie haben wir uns aber das so aufallend häufige symmetrische Erkranken eines Organes vorzustellen? Und dann den recht oft akuten Charakter derselben? Und weshalb erkranken gerade so häufig die Augen und die Kniegelenke? Wir sind gezwungen anzunehmen, daß von einem dritten Ort her Spirochaeten auf dem Wege der Blut- oder Lymphbahnen hierher gelangen. Vielleicht sogar kreisen die Spirochaeten auch in solchen Spätstadien der Syphilis öfter im Organismus, als wir bisher annehmen. Das Trauma spielt, wie aus den Anamnesen herrorgeht, bei dem Auftreten akuter Prozesse eine sehr große Rolle. Gerade Kniegelenksentzündungen entwickeln sich sehr häufig in Anschluß an eine Verletzung. Durch das Trauma wird ein Locus minoris resistentiae gesetzt. Als dieses Depot für die Spirochaeten müssen wir wohl die erkrankten Lymphdrüsen ansprechen, welche wir 
so häufig fanden. Hierüber würden weitere eingehende Untersuchungen AufschluB bringen.

Und wie kommt es, daB wir in diesen Fällen stets te rtiär syphilitische Produkte seheu und niemals solche Erscheinungen, welche der sog. Sekundärperiode zuzurechnen sind?

Gerade hierin liegt ja der Beweis, dab sich die kongenitale Syphilis in ihrem Verlauf durch nichts unterscheidet von der akquirierten, und daß wir nicht berechtigt sind, die Syphilis hereditaria tarda als ein Krankheitsbild sui generis zu behandeln.

Was leistet die Therapiebei der Lues bereditaria in ihren Spätformen?

Wenn man die vielen hierüber vorliegenden Mitteilungen durchliest, so fallen die günstigen Berichte auf. Abgesehen von einzelnen günstigen Erfahrungen können wir sie nicht ganz bestätigen. Allerdings bietet ja die Keratitis parenchymatosa eine verhältnismäßig bessere Prognose. Sie heilt in vielen Fällen unter antiluetischer Behandlung so aus, daff fast normale Sehschärfe wieder eintritt. Auch die Rezidive hierbei sind verhältnismäßig selten. Sehr viel ungünstiger ist dagegen die Prognose der Knochen- und Kniegelenkserkrankungen. Sie widerstehen auch einer zielbewußten $\mathrm{Hg}$-Behandlung und rezidivieren häufig. Gar nicht so sehr selten sahen wir auch spontane Besserungen und sogar Heilungen. Als die beste Behandlungsmethode hat sich uns eine über längere Zeit fortgesetzte Jodkalikur bewährt mit einer daran anschließenden $\mathrm{Hg}$-Kur. Es ist jedoch notwendig, derartige Kranke auf lange Zeit unter Beobachtung zu haiten, um im Falle eines Rezidivs sofort wieder behandeln zu können.

Wir haben in 3 Fällen Ehrlich 606 gegeben; in zwei Fällen ohne jeden Erfolg. Bei Fall 31 gingen die ausgedehnten Gummata so schnell zurïck, wie wir es mit $\mathrm{Hg}$ nicht erreicht hatten. Dieses sehr günstige Resultat ermutigt zur weiteren Anwendung. 
Es wäre im Sinne Welanders zu wünschen und zu hoffen, wenn durch Errichtung gleicher Kinderasyle wie das lilla hemmet das traurige Los der vielen kongenitalsyphilitischen Kinder wenigstens etwas günstiger und freundlicher gestaltet werden könnte. 